\title{
Subvalvular aortic stenosis: Comprehensive cardiac evaluation with dual-source computed tomography
}

Paul Stolzmann, MD, ${ }^{a}$ Hans Scheffel, MD, a Dominique Bettex, MD, ${ }^{b}$ Christoph Karlo, ${ }^{\text {a }}$ Thomas Frauenfelder, MD, ${ }^{a}$ René Prêtre, MD, ${ }^{\mathrm{c}}$ Borut Marincek, MD, and Hatem Alkadhi, MD, ${ }^{a}$ Zurich, Switzerland

Supplemental material is available online.

N

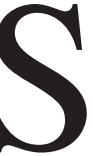

ubvalvular aortic stenosis (SAS) represents a fixed obstruction of the left ventricular outflow tract (LVOT) by an endocardial fold, fibrous tissue, or fibromuscular ridge. Echocardiography including color Doppler is the modality of choice to establish the diagnosis and to assess the extent and hemodynamic severity of SAS. ${ }^{1}$ We present imaging findings of a cardiac dual-source computed tomographic (CT) examination in a patient with SAS to demonstrate the potential of the noninvasive modality for a comprehensive evaluation of patients with the disease.

\footnotetext{
From the Institute of Diagnostic Radiology a and Institute of Anesthesia, Division of Cardiovascular Anesthesia, ${ }^{\mathrm{b}}$ and the Clinic for Cardiovascular Surgery, ${ }^{\mathrm{c}}$ University Hospital, Zurich, Switzerland.

This research has been supported by the National Center of Competence in Research, Computer Aided and Image Guided Medical Interventions of the Swiss National Science Foundation.

Received for publication Feb 27, 2007; accepted for publication March 8, 2007.

Address for reprints: Hatem Alkadhi, MD, Institute of Diagnostic Radiology, University Hospital Zurich, Raemistrasse 100, 8091 Zurich, Switzerland (E-mail: hatem.alkadhi@usz.ch).

J Thorac Cardiovasc Surg 2007;134:240-1

$0022-5223 / \$ 32.00$

Copyright $\odot 2007$ by The American Association for Thoracic Surgery doi:10.1016/j.jtcvs.2007.03.005
}

\section{Clinical Summary}

A 40-year-old woman was referred for follow-up after resection of a SAS 32 years ago. Clinical examination revealed a systolic grade 3/6 heart murmur and electrocardiography (ECG) showed stressinduced ST-segment depressions. Echocardiography demonstrated recurrence of the SAS causing LVOT obstruction (mean systolic pressure gradient $50 \mathrm{~mm} \mathrm{Hg}$ ). In addition, mild aortic regurgitation and prolapse of the anterior mitral valve leaflet were diagnosed. Thus, the patient was advised to undergo surgical resection of the SAS with aortic valve replacement.

Preoperative workup included a CT coronary angiographic examination for exclusion of coronary artery disease. The patient underwent retrospective ECG-gated dual-source CT (Definition, Siemens Medical Solutions, Erlangen, Germany) after intravenous administration of $65 \mathrm{~mL}$ of iodinated contrast medium (Ultravist 300; Schering, Berlin, Germany). CT accurately depicted the fibromuscular ridge with a muscular base at the crest of the interventricular septum causing significant narrowing of the LVOT (Figure 1). The tricuspid aortic valve showed homogeneous thickening of the cusps and a coaptation defect in mid-diastole indicating aortic regurgitation (Figure 2, A). In addition, billowing of the anterior mitral valve leaflet in early systole indicated mitral valve prolapse (Figure 2, B). Left ventricular myocardial mass was elevated (308 g, adjusted: $\left.178 \mathrm{~g} / \mathrm{m}^{2}\right)$, whereas end-systolic (21 $\mathrm{mL})$ and end-diastolic volume $(120 \mathrm{~mL})$ as well as ejection fraction $(83 \%)$ were within normal limits. The coronary artery tree could be depicted with diagnostic image quality and showed no significant coronary stenosis (Figure E1).

Subsequently, the patient was reoperated on by a modified Konno procedure. The SAS was resected and the aortic valve was replaced. The postoperative course was uneventful and the patient was discharged to a rehabilitation facility on the eleventh postoperative day.

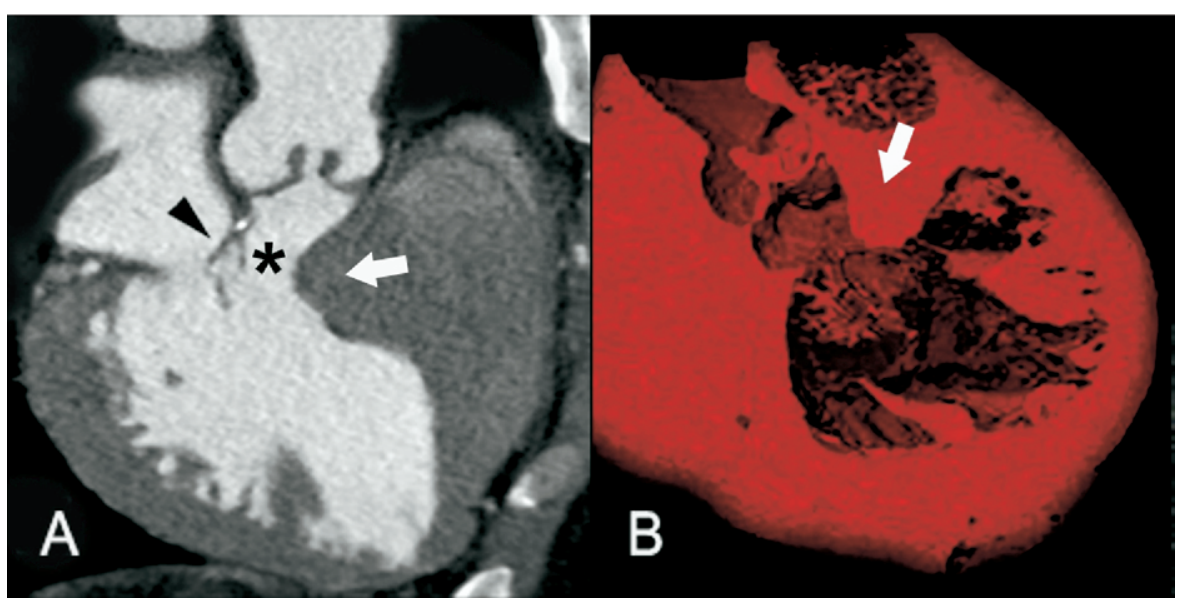

Figure 1. A, Dual-source computed tomographic coronary angiograph: Obliquecoronal reconstruction showing the fibromuscular bulge (arrow) originating from the interventricular septum causing significant narrowing of the LVOT (asterisk). Note the anterior mitral valve leaflet constituting the medial border of the LVOT (arrowhead). B, Three-dimensional volume-rendered image gives a better impression of the spatial relationship of the subaortic stenosis (arrow) and the obstruction of the LVOT. LVOT, left ventricular outflow tract. 


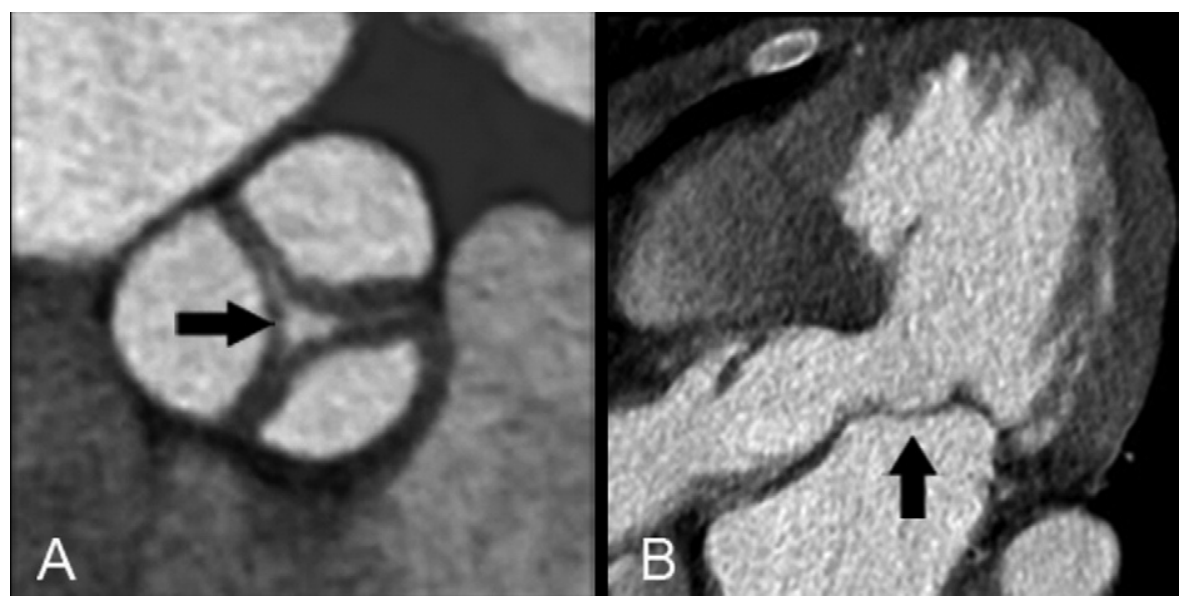

Figure 2. A, Dual-source computed tomographic coronary angiograph: Transverse reconstruction through the aortic valve in mid-diastole $(65 \%$ of the $R-R$ interval) demonstrates thickening of the aortic cusps and incomplete diastolic coaptation (arrow) leading to aortic regurgitation. $B$, Oblique-transverse reconstruction through the mitral valve in early systole ( $10 \%$ of the $\mathbf{R}-\mathbf{R}$ interval) shows billowing of the anterior leaflet (arrow) toward the left atrium, thus defining mitral valve prolapse.

\section{Discussion}

The peculiar anatomy of the LVOT formed by the anterior, septal, and posterior walls of the left ventricle and by the anterior leaflet of the mitral valve gives rise for various and complex variants of SAS. They may present as thin membrane consisting of an endocardial fold and/or fibrous tissue, as a fibromuscular ridge consisting of a thickened membrane with a muscular base at the crest of the interventricular septum, as a fibromuscular ring or collar that is circumferentially attached to the LVOT and to the base of the anterior mitral valve leaflet, or as a fibromuscular ridge causing tunnel-like narrowing of the LVOT.

Precise knowledge of SAS morphologic characteristics is a requisite for successful surgery, and the extent of SAS has been shown to be associated with postoperative remodeling of the LVOT complex. ${ }^{2}$ Our case study demonstrates that CT enables a comprehensive evaluation of the heart in a patient with SAS. CT provided accurate 3-dimensional morphoanatomic information about the fibromuscular bulge causing LVOT obstruction, allowed the characterization of additional valvular disorders that are often associated with SAS, enabled the quantification of left ventricular functional parameters, and at the same time reliably excluded coronary artery disease.

Whereas the clinical application of CT for noninvasive coronary angiography has widely emerged in past years, ${ }^{3}$ imaging of cardiac valvular disorders with CT has not yet gained broad acceptance. The reason for this might be the fact that CT is not supposed to provide hemodynamic measures by being a purely morphology-based imaging tool. On the other hand, several recent investigations have shown that $\mathrm{CT}$ also allows the quantification of severity of valvular heart disease by planimetric measurements of the regurgitant orifice area for assessing the degree of aortic regurgitation ${ }^{4}$ and by planimetric measurements of the aortic valve area for grading the severity of aortic stenosis. ${ }^{5}$
The recently introduced dual-source CT scanner technology has further advanced the capability of the modality for cardiac imaging. This scanner type is characterized by two x-ray tubes and two corresponding 64-slice detectors mounted onto the rotating gantry with an angular offset of $90^{\circ}$. This configuration allows for ECG-gated cardiac imaging with a high temporal resolution of 83 $\mathrm{ms}$, irrespective of the heart rate of the patient, that in future may enable imaging not only of coronary arteries at higher heart rates but also of rapidly moving cardiac valves, especially in systole.

In conclusion, dual-source CT accurately depicts valvular and subvalvular cardiac disease, providing excellent morphologic anatomy, and at the same time allows the reliable exclusion of coronary artery disease. This information may prove useful for preoperative planning of cardiac surgery.

\section{References}

1. Sigfusson G, Tacy TA, Vanauker MD, Cape EG. Abnormalities of the left ventricular outflow tract associated with discrete subaortic stenosis in children: an echocardiographic study. J Am Coll Cardiol. 1997;30: 255-9.

2. Barkhordarian R, Wen-Hong D, Li W, Josen M, Henein M, Ho SY. Geometry of the left ventricular outflow tract in fixed subaortic stenosis and intact ventricular septum: an echocardiographic study in children and adults. $J$ Thorac Cardiovasc Surg. 2007;133:196-203.

3. Scheffel H, Alkadhi H, Plass A, Vachenauer R, Desbiolles L, Gaemperli $\mathrm{O}$, et al. Accuracy of dual-source CT coronary angiography: first experience in a high pre-test probability population without heart rate control. Eur Radiol. 2006;16:2739-47.

4. Feuchtner GM, Dichtl W, Schachner T, Muller S, Mallouhi A, Friedrich GJ, et al. Diagnostic performance of MDCT for detecting aortic valve regurgitation. AJR Am J Roentgenol. 2006;186:1676-81.

5. Alkadhi H, Wildermuth S, Plass A, Bettex D, Baumert B, Leschka S, et al. Aortic stenosis: comparative evaluation of 16-detector row $\mathrm{CT}$ and echocardiography. Radiology. 2006;240:47-55. 


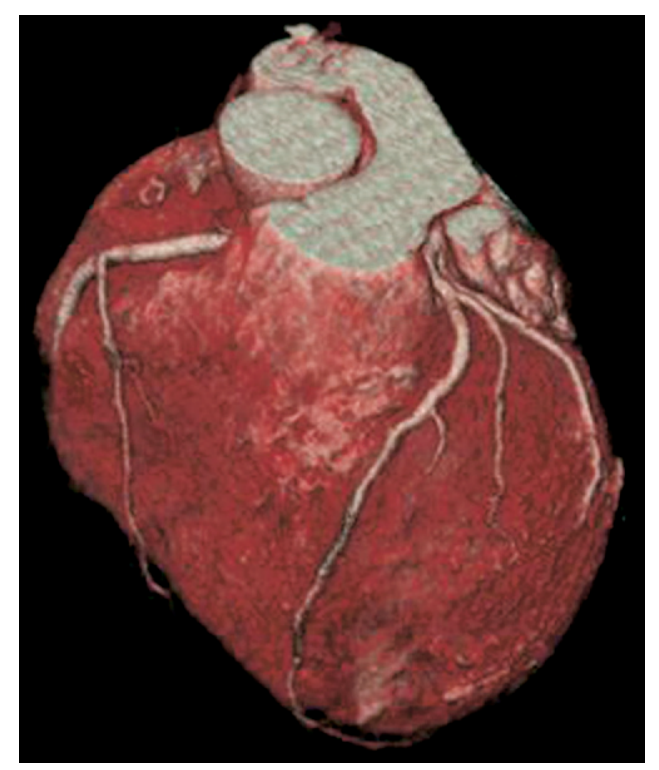

Figure E1. Three-dimensional volume rendered image of the heart demonstrating the coronary artery tree with no atherosclerotic changes. 